\title{
Sarcoma metastasis to the pancreas: experience at a single institution
}

\author{
Miseon Lee ${ }^{1}$, Joon Seon Song ${ }^{1}$, Seung-Mo Hong ${ }^{1}$, Se Jin Jang ${ }^{1}$, Jihun Kim ${ }^{1}$, Ki Byung Song ${ }^{2}$, Jae Hoon Lee ${ }^{2}$, Kyung-Ja Cho ${ }^{1}$ \\ ${ }^{1}$ Department of Pathology, 2Division of Hepatobiliary and Pancreatic Surgery, Department of Surgery, Asan Medical Center, University of Ulsan College of Medicine, Seoul, Korea
}

\begin{abstract}
Background: Reports of metastatic sarcoma to the pancreas are limited. We reviewed the clinicopathologic characteristics of such cases. Methods: We reviewed 124 cases of metastatic tumors to the pancreas diagnosed at Asan Medical Center between 2000 and 2017. Results: Metastatic tumors to the pancreas consisted of 111 carcinomas (89.5\%), 12 sarcomas (9.6\%), and one melanoma (0.8\%). Primary sarcoma sites were bone $(n=4)$; brain, lung, and soft tissue $(n=2$ for each); and the uterus and pulmonary vein $(n=1$ for each). Pathologically, the 12 sarcomas comprised 2 World Health Organization grade III solitary fibrous tumors/hemangiopericytomas, and one case each of synovial sarcoma, malignant solitary fibrous tumor, undifferentiated pleomorphic sarcoma, osteosarcoma, mesenchymal chondrosarcoma, intimal sarcoma, myxofibrosarcoma, myxoid liposarcoma, rhabdomyosarcoma, subtype uncertain, and high-grade spindle-cell sarcoma of uncertain type. The median interval between primary cancer diagnosis and pancreatic metastasis was 28.5 months. One case manifested as a solitary pancreatic osteosarcoma metastasis 15 months prior to detection of osteosarcoma in the femur and was initially misdiagnosed as sarcomatoid carcinoma of the pancreas. Conclusions: The metastatic sarcoma should remain a differential diagnosis when spindle-cell malignancy is found in the pancreas, even for solitary lesions or in patients without prior history.
\end{abstract}

Key Words: Pancreas; Metastasis; Sarcoma

Received: January 20, 2020 Revised: February 26, 2020 Accepted: March 4, 2020

Corresponding Author: Kyung-Ja Cho, MD, PhD, Department of Pathology, Asan Medical Center, University of Ulsan College of Medicine, 88 Olympic-ro 43-gil, Songpa-gu, Seoul 05505, Korea

Tel: +82-2-3010-4545, Fax: +82-2-472-7898, E-mail: kj@@amc.seoul.kr

Most pancreatic tumors are primary, and the majority are adenocarcinomas of ductal origin [1,2]. Although direct invasion of the pancreas by adjacent aggressive tumors is often observed, pancreatic metastasis from distant primary sites is very rare $[1,3]$. Metastatic pancreatic cancer accounts for less than $2 \%$ of all pancreatic malignancies $[4,5]$. Renal cell carcinoma is the most common primary tumor to metastasize to the pancreas $[4,5]$. Other common tumors are colorectal cancer, melanoma, breast cancer, lung cancer, and sarcoma [4]. Among these, cases of metastatic sarcoma to the pancreas are limited [4]. Metastatic sarcoma to the pancreas may present a diagnostic and therapeutic challenge due to its rarity and the difficulty in distinguishing between primary and metastatic tumors based on their radiologic features, especially for solitary mass [3,6]. Furthermore, tumors located in the body or tail of the pancreas often present with no symptoms $[3,7]$. Therapeutic approaches can vary greatly between primary pancreatic cancer and metastatic sarcoma [3,4,8-10]. With continued improvements in treatment and survival for sarcoma patients, the frequency of detection of metastasis to unusual sites is increasing [11]. However, the index of suspicion for metastatic sarcoma in the pancreas is still very low, and a standard management regimen for metastatic pancreatic sarcoma has not been established. Prolonged survival after surgery for isolated or resectable pancreatic metastatic sarcoma has been reported [10,12-16].

In this paper, we present 12 cases of metastatic sarcoma to the pancreas and report their clinical and histologic features.

\section{MATERIALS AND METHODS}

One hundred twenty-four cases of metastasis to the pancreas diagnosed by biopsy $(n=49,39.5 \%)$ or surgical resection $(n=$ 75, 60.5\%) at Asan Medical Center between 2000 and 2017 were reviewed. Only distant metastatic tumors to the pancreas were included; adjacent tumors directly extending to the pancreas were excluded. Patient medical records were retrospectively reviewed to evaluate clinical presentation, treatment, and patient status. Hematoxylin and eosin-stained slides of both 
primary tumors and pancreatic metastases were reviewed by two pathologists (M.L. and K.J.C.).

\section{Ethics statement}

This study was approved by the appropriate institutional review board (2020-0048), and the informed consent was waived.

\section{RESULTS}

The primary tumors of 124 patients with pancreatic metastases included 111 carcinomas (89.5\%), 12 sarcomas (9.6\%), and one melanoma $(0.8 \%)$. The metastatic carcinomas $(\mathrm{n}=111)$ were mainly renal cell carcinomas ( $n=50,40.3 \%)$, followed by lung cancer $(\mathrm{n}=18$; eight small-cell carcinomas, five adenocarcinomas, three squamous cell carcinomas, and two sarcomatoid carcinomas), colorectal cancer ( $\mathrm{n}=12$, all adenocarcinomas), gastric cancer $(n=12$; six tubular adenocarcinomas, three poorly cohesive carcinomas, two mucinous adenocarcinomas, and one hepatoid adenocarcinoma), ovarian or fallopian tube cancers ( $\mathrm{n}=$ 7; six serous carcinomas and one clear-cell carcinoma), hepatocellular carcinoma $(n=4)$, and other cancers $(n=8)$.

Of the 12 sarcoma patients, nine were female and three were male, with ages ranging from $20-70$ years (Table 1). Among the primary lesions, the most common site was bone $(n=4)$; followed by brain, lung, soft tissue ( $\mathrm{n}=2$ for each), uterus, and pulmonary vein ( $\mathrm{n}=1$ each) (Table 1). Histologically, the sarcomas included two World Health Organization (WHO) grade III solitary fibrous tumors/hemangiopericytomas, and one case each of synovial sarcoma, malignant solitary fibrous tumor, undifferentiated pleomorphic sarcoma, osteosarcoma, mesenchymal chondrosarcoma, intimal sarcoma, myxofibrosarcoma, myxoid liposarcoma, rhabdomyosarcoma, subtype uncertain, and high-grade spindle-cell sarcoma of uncertain type (Table 1). In the case of synovial sarcoma, the presence of an SYT-SSX fusion product was confirmed by reverse transcription polymerase chain reaction.

The primary tumor of case no. 8 arose in the medullary cavity of the metaphysis of the distal femur. The tumor destroyed the cortex and extended into the adjacent soft tissue. Clinically, osteosarcoma was highly suspected, but neoplastic bone formation, which is the essential diagnostic feature of osteosarcoma, was not observed in either primary or metastatic tumor. Thus, we diagnosed it as a high-grade spindle-cell sarcoma of uncertain type.

The median interval between primary sarcoma diagnosis and detection of pancreatic metastasis was 28.5 months. In one exceptional case, a pancreatic metastasis of an osteosarcoma mani- fested 15 months prior to detection of the osteosarcoma in the femur (Table 1). This patient first presented with abdominal pain, and abdominal computed tomography revealed a $2.5-\mathrm{cm}$ mass-like lesion in the pancreatic tail. Other radiologic workup, such as imaging of the extremities, was not performed at that time. A $2.7-\mathrm{cm}$, ovoid, solid mass was identified during distal pancreatectomy (Fig. 1A). Microscopy revealed proliferation of pleomorphic spindle cells, numerous giant cells with infiltrative margins (Fig. 1B), high mitotic activity, and focal osteoid and bone formation (Fig. 1C). Vascular invasion was also identified (Fig. 1D). The tumor was diagnosed as a sarcomatoid carcinoma of the pancreas. The patient was hospitalized with left leg pain 15 months after distal pancreatectomy. Magnetic resonance imaging revealed a 9-cm mass in the left femur with fracture and adjacent soft-tissue extension (Fig. 1E). A biopsy was performed, revealing histologic features similar to those of the pancreatic mass (Fig. 1F). We concluded that the osteosarcoma of the femur had metastasized to the pancreas, and the original diagnosis of sarcomatoid carcinoma of the pancreas was incorrect.

Metastatic tumors were located in the body or tail of the pancreas $(n=8)$, the head of the pancreas $(n=3)$, and throughout the entire pancreas $(n=1)$ (Table 1). Nine cases were single masses, and three cases showed multiple masses. Pancreatic tumor size ranged from 2.5-19 cm (median, $2.7 \mathrm{~cm}$ ) (Table 1). Primary lesions and pancreatic metastatic lesions (Fig. 2) showed similar histologic features except in one case in which about $30 \%$ of the round-cell components were present in the primary myxoid liposarcoma but not in the metastasis.

Primary tumors from 12 patients had undergone surgical resection: two patients with distant metastasis synchronously received neoadjuvant chemotherapy, six received postoperative or preoperative radiation therapy, and four received adjuvant chemotherapy (Table 1). The chemotherapy regimens varied, and several combinations of methotrexate, ifosfamide, doxorubicin, cisplatin, and etoposide were used. In five patients, local recurrence of the primary lesions occurred prior to detection of pancreatic metastasis (Table 1).

After diagnosis of metastatic pancreatic lesions, nine of 12 patients underwent surgical resection with or without preoperative chemotherapy or radiation therapy. Five cases received postoperative radiation therapy or chemotherapy (Table 1). Lymphnode dissection was performed in eight cases, and none of the cases showed metastasis to the lymph nodes. After detection of pancreatic metastasis, eight patients died between 5-48 months, and four patients remained alive from 4-92 months (Table 1). 


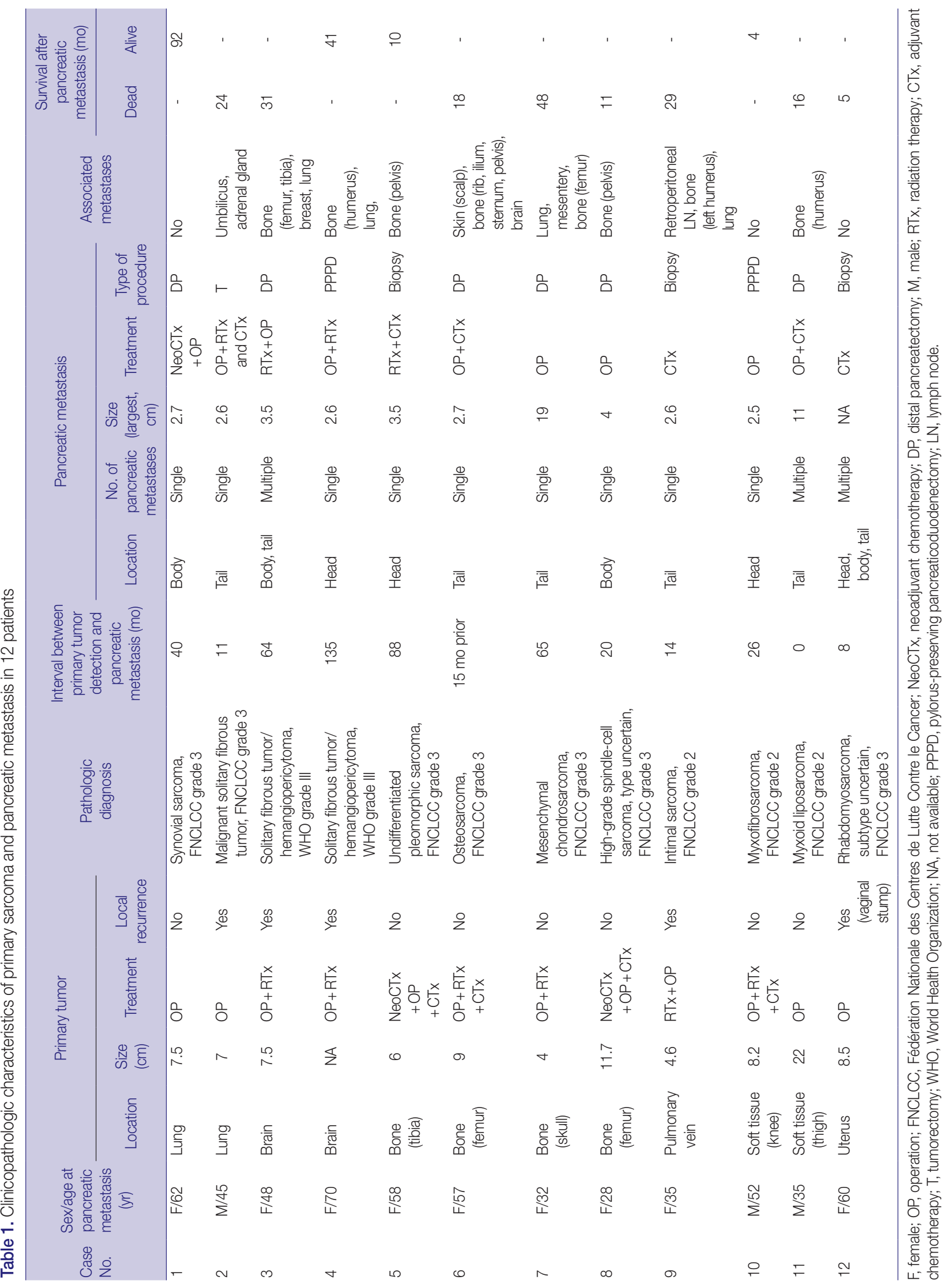



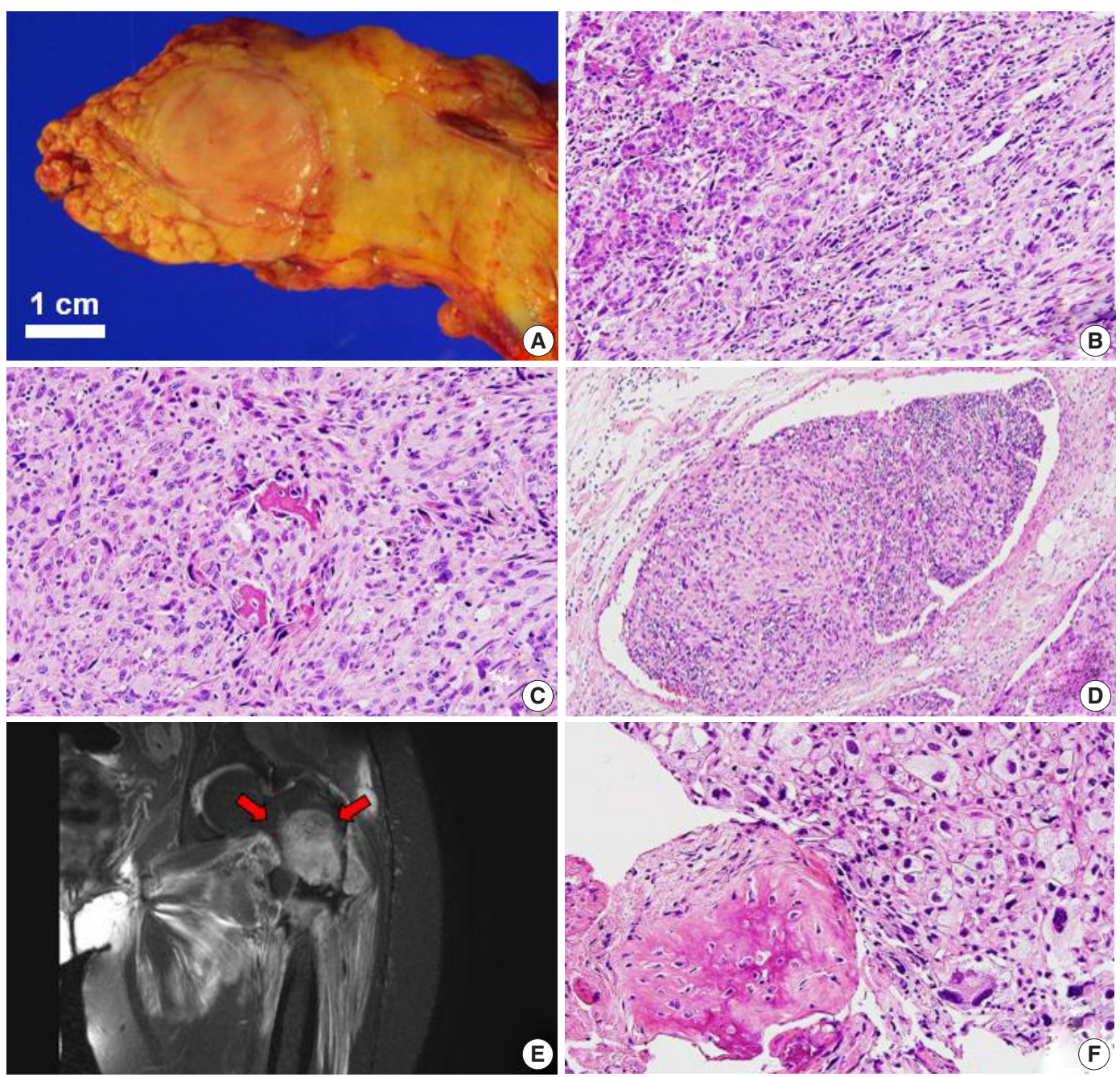

Fig. 1. Primary (A-D) and metastatic (E, F) osteosarcoma. (A) Gross photographs of a distal pancreatectomy specimen bisected along the main pancreatic duct. A well-defined 2.7-cm ovoid solid mass is observed in the tail of the pancreas. Metastatic osteosarcoma to the pancreas shows infiltrative margins (B) and consists of pleomorphic cells and multinucleated giant cells with bone formation and osteoid stroma (C). (D) Vascular invasion is noted. (E) T2-weighted coronal magnetic resonance imaging showing an approximately 9-cm soft tissue mass in the left femur (arrows). (F) The primary lesion of the femur shows similar features to the metastatic pancreatic lesion.

\section{DISCUSSION}

Sarcomas preferentially metastasize via the blood rather than the lymphatic system, and lung and bone tissue are the most frequent sites of metastatic sarcoma [17]. Lymph node metastasis is uncommon, but some histologic sarcoma types exhibit high metastasis rates via lymphatic drainage, including rhabdomyosarcoma, angiosarcoma, clear-cell sarcoma, epithelioid sarcoma, and myxoid liposarcoma $[18,19]$. In our study, the absence of lymph node metastasis in all eight lymph node dissection cases suggests that metastatic sarcoma to the pancreas is more likely to occur via the blood than the lymphatic system. The connective interspaces and cavities such as the peritoneum or pleural space, in which tumor cells can become entrapped, are also possible routes of metastatic dissemination [18].

Synovial sarcoma metastasizes in approximately $50 \%$ of cases, and common metastatic sites are the lung and bone [12]. Unlike other sarcomas, spread to the lymph nodes is not infrequent (3\%-23\%) [12]. For disseminated disease, chemotherapy based on adriamycin and ifosfamide is usually attempted. Makino et al. [12] suggested that pancreatic metastasis from synovial sarcoma can be successfully treated by surgical resection in cases of solitary pancreatic lesions with no extra-pancreatic metastasis and a more than 3-year interval between diagnosis of the primary tumor and pancreatic metastasis. Only four cases of metastatic synovial sarcoma to the pancreas have been reported in 
the literature $[5,10,12,20]$, making our case the fifth. The patient reported here was also treated by surgical resection. Our patient and the two patients described by Makino et al. [12] are doing well, without recurrence or distant metastasis for 92 months, 40 months, and 30 months after surgical resection, respectively.
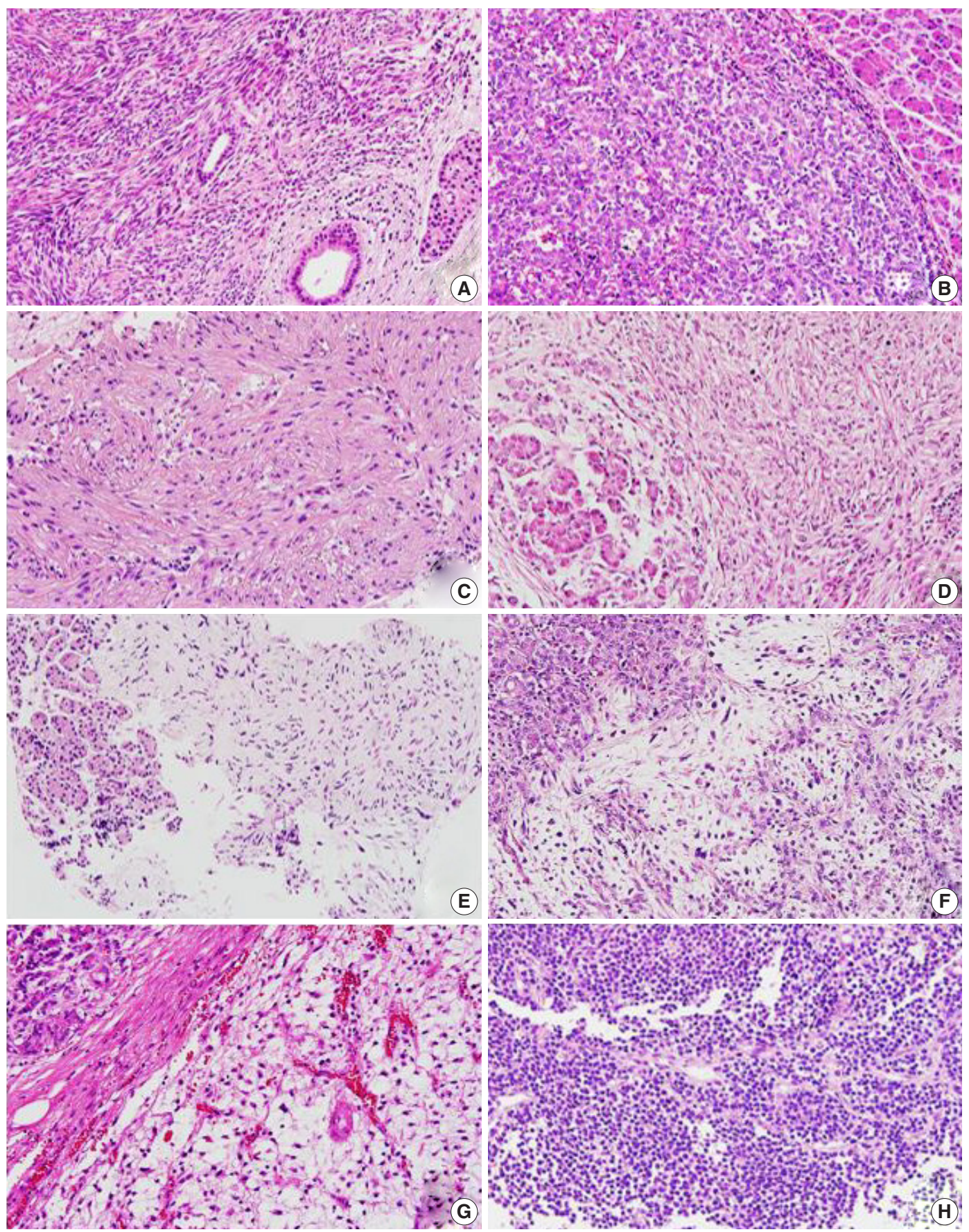

Fig. 2. Metastatic sarcomas to the pancreas. (A) Synovial sarcoma from the lung with cellular fascicles of uniform spindle cells. (B) World Health Organization grade III solitary fibrous tumor/hemangiopericytoma from the brain composed of spindle cells with prominent vascular structure. (C) Undifferentiated pleomorphic sarcoma from the tibia with fascicular arrangement of atypical spindle cells. (D) High-grade spindle-cell sarcoma from the femur. (E) Intimal sarcoma from the pulmonary vein. (F) Myxofibrosarcoma from the knee showing prominent myxoid stroma and elongated, thin-walled blood vessels. (G) Myxoid liposarcoma from the thigh with abundant myxoid stroma, a delicate, arborizing capillary vasculature, and surrounding lipoblasts. (H) Rhabdomyosarcoma, subtype uncertain, from the uterus showing scattered eosinophilic rhabdomyoblasts. 
Solitary fibrous tumors/hemangiopericytomas of the central nervous system (CNS) show two distinct histologic phenotypes, a solitary fibrous tumor phenotype and a hemangiopericytoma phenotype [21]. The hemangiopericytoma phenotype has a high recurrence rate $(75 \%-90 \%)$ and a high rate of distant metastasis (20\%-33\%), especially to the liver, lung, and bone [14,21-24]. Both our cases showed the hemangiopericytoma phenotype and were grade III. The metastatic sites of these cases were the lung, bone, breast, and pancreas. A total of 18 cases of metastasis from the brain to the pancreas have been reported to date $[14,25]$. Interestingly, the interval between diagnosis of the primary tumor and development of pancreas metastasis was long (5.3-24 years, with a median of 10.13 years) [14,25]. After detection of pancreatic metastasis, eight patients died between 2.6-16 years, and seven patients lived for 3.4-25 years.

Outside the CNS, such as in the soft tissue, pleura, and other visceral sites, malignant solitary fibrous tumors showed increased cellularity and mitotic activity (> 4 per 10 high-powered fields). Metastatic malignant solitary fibrous tumors to the pancreas have been reported in two cases, one originating in the chest wall [13] and one originating in the kidney [26]. Our case originated in the lung.

Osteosarcoma has a high potential for metastasis [27]. The high incidence of metastasis is related to its strong propensity for early hematogenous spread $[15,16,28]$. Prolonged survival is possible with treatment including multi-centric chemotherapy and resection of the primary tumor $[3,11,15,28]$. This result is attributed to the delayed appearance of metastasis and increased metastasis incidence at unusual sites [11]. The common metastasis sites are the lung, bone, pleura, and liver [3,27,29]. Twelve cases of metastatic pancreatic osteosarcoma have been reported [3,6,11,15,16,27-32], making our case the thirteenth. The median interval between primary osteosarcoma diagnosis and development of pancreas metastasis was three years (range, -1.25 to 11 years), including our case, which was the first reported case with a pancreatic metastasis diagnosis prior to primary osteosarcoma diagnosis. Among the eight patients with available follow-up information, four who had undergone surgical resection of the pancreatic metastasis were alive for $7-13$ months. Three patients died two weeks, three months, and 18 months after pancreatic metastasis development, respectively [3,28,29,32].

Mesenchymal chondrosarcoma mainly metastasizes to the lungs [33]. Metastasis to the pancreas is extremely rare and has only been reported in six cases, making our case the seventh [1,10,33-36]. The primary site was extra-skeletal soft tissue (thigh, brain, buttock, or femoral vein) in five cases and bone (skull) in two cases. Among the four patients with available follow-up information, two were alive 6 years and 10 years after surgery for pancreatic metastasis, and two patients died 2 and 4 years after surgery.

Myxoid liposarcoma generally has a favorable prognosis. The round-cell content is a poor prognostic factor in this disease. The most common metastatic site is the abdomen, including the retroperitoneum, abdominal wall, and abdominal cavity, followed by bone $[37,38]$. Until now, metastasis to the pancreas has not been reported. Our case showed metastasis from the thigh to the bone and the pancreas. Microscopy showed roundcell content of approximately $30 \%$ in the primary lesion, but not in the metastatic lesion.

Myxofibrosarcoma was first described in 1977 as a myxoid variant of malignant fibrous histiocytoma and was reclassified as myxofibrosarcoma in 2013 by the WHO [39]. The most common metastatic site is the lung, followed by the pleura, lymph node, bone, and retroperitoneum [40,41]. In this report, a case of myxofibrosarcoma in the knee showed metastasis to the pancreas. Only two cases of pancreatic metastasis of a myxofibrosarcoma have been previously reported, although there are some papers that present cases of metastatic myxofibrosarcoma without describing the specific metastatic sites [40]. Some studies show that adjuvant or neoadjuvant radiation therapy may improve recurrence and metastasis in this indication.

In this study, we present several rare cases of metastatic sarcomas to the pancreas. This is the largest reported case series of pancreatic metastatic sarcomas to date. Metastatic sarcoma comprised $10 \%$ of pancreatic metastases, which is high given the overall sarcoma incidence. Furthermore, pancreatic metastasis may manifest as a primary solitary lesion before detection of the primary tumor. Intensive whole-body imaging screening is necessary in sarcoma patients, and metastasis should remain a differential diagnosis in pancreatic tumors with sarcomatoid features. Recently, the efficacy of surgical resection of pancreatic metastases was shown for sarcoma [10,12-16] as well as renal cell carcinoma [4]. Clinicians caring for patients with sarcoma should be aware of the potential for increased survival following surgical resection of the metastasis. This study will contribute to a better understanding of this unusual clinical circumstance and, thus, may lead to improved diagnostic accuracy and treatment.

\section{ORCID}

Miseon Lee: https://orcid.org/0000-0002-6385-7621

Joon Seon Song: https://orcid.org/0000-0002-7429-4254 
Seung-Mo Hong: https://orcid.org/0000-0002-8888-6007

Se Jin Jang: https://orcid.org/0000-0001-8239-4362

Jihun Kim: https://orcid.org/0000-0002-8694-4365

Ki Byung Song: https://orcid.org/0000-0001-5422-5481

Jae Hoon Lee: https://orcid.org/0000-0002-6170-8729

Kyung-Ja Cho: https://orcid.org/0000-0002-4911-7774

\section{Author Contributions}

Conceptualization: KJC.

Data curation: ML, KJC.

Formal analysis: ML, KJC.

Funding acquisition: KJC, SMH.

Investigation: ML, KJC.

Methodology: ML, KJC.

Project administration: ML.

Resources: JSS, SMH, SJJ, JK, KBS, JHL, KJC.

Supervision: KJC.

Validation: ML, KJC.

Visualization: ML.

Writing—original draft: ML, KJC.

Writing_-review \& editing: ML, KJC.

\section{Conflicts of Interest}

J.S.S. and S.J.J., contributing editors of the Journal of Pathology and Translational Medicine, were not involved in the editorial evaluation or decision to publish this article. All remaining authors have declared no conflicts of interest.

\section{Funding}

No funding to declare.

\section{REFERENCES}

1. Chatzipantelis P, Karvouni E, Fragoulidis GP, Voros D, Pafiti A. Clinicopathologic features of two rare cases of mesenchymal metastatic tumors in the pancreas: review of the literature. Pancreas 2006; 33: 301-3.

2. Adsay NV, Andea A, Basturk O, Kilinc N, Nassar H, Cheng JD. Secondary tumors of the pancreas: an analysis of a surgical and autopsy database and review of the literature. Virchows Arch 2004; 444: 527-35.

3. Bertucci F, Araujo J, Giovannini M. Pancreatic metastasis from osteosarcoma and Ewing sarcoma: literature review. Scand J Gastroenterol 2013; 48: 4-8.

4. Sperti C, Moletta L, Patanè G. Metastatic tumors to the pancreas: the role of surgery. World J Gastrointest Oncol 2014; 6: 381-92.
5. Krishna SG, Rao BB, Lee JH. Endoscopic sonography and sonographically guided fine-needle aspiration biopsy in the diagnosis of unusual pancreatic metastases from synovial sarcoma. J Clin Ultrasound 2014; 42: 180-2.

6. Lasithiotakis K, Petrakis I, Georgiadis G, Paraskakis S, Chalkiadakis G, Chrysos E. Pancreatic resection for metastasis to the pancreas from colon and lung cancer, and osteosarcoma. JOP 2010; 11: 593-6.

7. Shinagawa Y, Suzuki T, Hamanaka Y, Nishihara K, Takahasi M. Solitary pancreatic metastasis of malignant fibrous histiocytoma treated by distal pancreatectomy. Pancreas 1992; 7: 726-30.

8. Sperti C, Pasquali C, Piccoli A, Pedrazzoli S. Recurrence after resection for ductal adenocarcinoma of the pancreas. World J Surg 1997; 21: $195-200$.

9. Yeo CJ, Cameron JL, Sohn TA, et al. Six hundred fifty consecutive pancreaticoduodenectomies in the 1990s: pathology, complications, and outcomes. Ann Surg 1997; 226: 248-57.

10. Yamamoto H, Watanabe K, Nagata M, et al. Surgical treatment for pancreatic metastasis from soft-tissue sarcoma: report of two cases. Am J Clin Oncol 2001; 24: 198-200.

11. Aarvold A, Bann S, Giblin V, Wotherspoon A, Mudan SS. Osteosarcoma metastasising to the duodenum and pancreas. J Bone Joint Surg Br 2007; 89: 542-4.

12. Makino Y, Shigekawa M, Kegasawa T, et al. A case report of pancreatic metastasis from synovial sarcoma successfully treated by metastasectomy with adjuvant chemotherapy. Medicine (Baltimore) 2016; 95: e4789.

13. Colvin JS, Morris-Stiff G, Cruise M, Purysko A. Pancreatic metastasis from an osseous solitary fibrous tumour. BMJ Case Rep 2017; 2017: bcr-2017-220114.

14. Hiraide T, Sakaguchi T, Shibasaki Y, et al. Pancreatic metastases of cerebellar hemangiopericytoma occurring 24 years after initial presentation: report of a case. Surg Today 2014; 44: 558-63.

15. Rubin E, Dunham WK, Stanley RJ. Pancreatic metastases in bone sarcomas: CT demonstration. J Comput Assist Tomogr 1985; 9: 886-8.

16. Kim SJ, Choi JA, Lee SH, et al. Imaging findings of extrapulmonary metastases of osteosarcoma. Clin Imaging 2004; 28: 291-300.

17. Leong SP, Cady B, Jablons DM, et al. Clinical patterns of metastasis. Cancer Metastasis Rev 2006; 25: 221-32.

18. Pennacchioli E, Tosti G, Barberis M, et al. Sarcoma spreads primarily through the vascular system: are there biomarkers associated with vascular spread? Clin Exp Metastasis 2012; 29: 757-73.

19. Jacobs AJ, Morris CD, Levin AS. Synovial sarcoma is not associated with a higher risk of lymph node metastasis compared with other soft tissue sarcomas. Clin Orthop Relat Res 2018; 476: 589-98.

20. Patel S, Martins N, Yantis R, Shepro D, Levey J, Patwardhan R. Endoscopic management of metastatic synovial sarcoma to the pan- 
creas. Pancreas 2006; 33: 205-6.

21. Louis DN, Ohgaki H, Wiestler OD, Cavenee WK. WHO classification of tumours of the central nervous system. 4th ed. Lyon: International Agency for Research on Cancer, 2016; 249-54.

22. Enzinger FM, Smith BH. Hemangiopericytoma: an analysis of 106 cases. Hum Pathol 1976; 7: 61-82.

23. McMaster MJ, Soule EH, Ivins JC. Hemangiopericytoma: a clinicopathologic study and long-term followup of 60 patients. Cancer 1975; 36: 2232-44.

24. Teh BS, Lu HH, Jhala DN, Shahab I, Lynch GR. Pancreatic head mass from metastatic meningeal hemangiopericytoma. Sarcoma 2000; 4: 169-72.

25. Osuga T, Hayashi T, Ishiwatari $H$, et al. Pancreatic metastasis from a solitary fibrous tumor of the central nervous system. JOP 2014; 15: 58-62

26. Patel YA, Dhalla S, Olson MT, Lennon AM, Khashab MA, Singh VK. Pancreatic metastasis from a solitary fibrous tumor of the kidney: a rare cause of acute recurrent pancreatitis. Pancreatology 2013; 13: 631-3.

27. Avcu S, Akdeniz H, Arslan H, Toprak N, Unal O. A case of primary vertebral osteosarcoma metastasizing to pancreas. JOP 2009; 10: $438-40$.

28. Akpinar B, Obuch J, Fukami N, Pokharel SS. Unusual presentation of a pancreatic cyst resulting from osteosarcoma metastasis. World J Gastroenterol 2015; 21: 8452-7.

29. Jin P, Wang W, Su H, Sheng JQ. Osteosarcoma metastasizing to pancreas confirmed by endoscopic ultrasound-guided fine-needle aspiration. Endoscopy 2014; 46 Suppl 1 UCTN: E109-10.

30. Glass RJ, Eftekhari F, Kleinerman ES, Jaffe N, Nachman J. Osteosarcoma metastatic to the pancreas in young patients. Clin Radiol 1996; 51: 293-4.

31. Khan AS, Crowe DR, Trevino JM, Eloubeidi MA. Multiple metastases to the pancreas from primary maxillary osteosarcoma: diagno- sis with EUS-guided FNA. Gastrointest Endosc 2011; 73: 1320-2.

32. Toyama H, Asari S, Goto T, et al. A case of pancreatic metastasis of osteosarcoma resected using laparoscopic spleen preserving distal pancreatectomy. Gan To Kagaku Ryoho 2016; 43: 1988-90.

33. Guo J, Gu Y, Guo L, et al. A case of mesenchymal chondrosarcoma arising from the femoral vein with 8 years of follow-up. Ann Vasc Surg 2015; 29: 1455.

34. Tsukamoto S, Honoki K, Kido A, et al. Chemotherapy improved prognosis of mesenchymal chondrosarcoma with rare metastasis to the pancreas. Case Rep Oncol Med 2014; 2014: 249757.

35. Lightenstein L, Bernstein D. Unusual benign and malignant chondroid tumors of bone. A survey of some mesenchymal cartilage tumors and malignant chondroblastic tumors, including a few multicentric ones, as well as many atypical benign chondroblastomas and chondromyxoid fibromas. Cancer 1959; 12: 1142-57.

36. Komatsu T, Taira S, Matsui O, Takashima T, Note M, Fujita H. A case of ruptured mesenchymal chondrosarcoma of the pancreas. Radiat Med 1999; 17: 239-41.

37. Lin S, Gan Z, Han K, Yao Y, Min D. Metastasis of myxoid liposarcoma to fat-bearing areas: A case report of unusual metastatic sites and a hypothesis. Oncol Lett 2015; 10: 2543-6.

38. Estourgie SH, Nielsen GP, Ott MJ. Metastatic patterns of extremity myxoid liposarcoma and their outcome. J Surg Oncol 2002; 80: 8993.

39. Fletcher CD, Bridge JA, Hogendoorn P, Mertens F. WHO classification of tumours of soft tissue and bone. 4th ed. Lyon: International Agency For Research on Cancer, 2013; 93-5.

40. Hambleton C, Noureldine S, Gill F, Moroz K, Kandil E. Myxofibrosarcoma with metastasis to the lungs, pleura, and mediastinum: a case report and review of literature. Int J Clin Exp Med 2012; 5: 92-5.

41. Neagu TP, Sinescu RD, Enache V, Achim SC, Tiglis M, Mirea LE. Metastatic high-grade myxofibrosarcoma: review of a clinical case. Rom J Morphol Embryol 2017; 58: 603-9. 